\title{
Hernia of Morgagni: Case report
}

\author{
Jared M. Huston, MD, Heather King, MD, Alison Maresh, MD, David Liska, MD, \\ Jeffrey L. Port, MD, Nasser K. Altorki, MD, and Paul C. Lee, MD, New York, NY
}

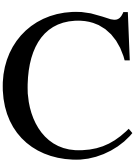

ongenital diaphragmatic hernias are relatively rare, occurring in $0.02 \%$ to $0.05 \%$ of live births. ${ }^{1}$ Morgagni hernia is the least common type of congenital diaphragmatic hernia and is often diagnosed incidentally in asymptomatic adults. ${ }^{2,3}$ We present the case of a symptomatic 70 year-old woman with a large Morgagni hernia. She underwent primary hernia repair without mesh via an upper midline laparotomy. Her postoperative course was uncomplicated, and she was well at the 1-month follow-up.

\section{Clinical Summary}

A 70-year-old woman was found to have an abnormality on a chest radiogram before bladder surgery 10 years prior. A follow-up computed tomographic scan demonstrated a large, right-sided anterior diaphragmatic hernia of the Morgagni type, along with fibrotic and atelectatic changes of the right lung (Figure 1). The patient reported symptoms of dyspepsia, epigastric pain, and dyspnea on exertion for several years.

The patient was brought to the operating room and intubated using a double-lumen endotracheal tube in the event a thoracotomy became necessary. She underwent an upper midline laparotomy and abdominal exploration, which revealed a right-sided, 6-cm anteromedial diaphragmatic defect. No other anatomic defects were noted. After lysis of adhesions, the borders of the hernia defect were identified, and the hernia sac was dissected free using electrocautery. The hernia contents were then reduced with gentle traction. The hernia sac contained the entire transverse colon and greater omentum (Figure 2). The colon was inspected and found to be without signs of ischemia or obstruction. The hernia sac was then excised completely. The right lung was ventilated and found to expand inferiorly down to the diaphragm. A $28 \mathrm{~F}$ chest tube was placed. The diaphragmatic defect was then reapproximated without tension using interrupted, polypropylene sutures (Prolene; Ethicon, Inc, Somerville,

From the Division of Thoracic Surgery, Department of Cardiothoracic Surgery, New York-Presbyterian Hospital/Weill Medical College of Cornell University, New York, NY.

Received for publication July 12, 2007; accepted for publication Sept 21, 2007.

Address for reprints: Jared M. Huston, MD, Weill Medical College of Cornell University, New York Presbyterian Hospital, Division of Thoracic Surgery, Department of Cardiothoracic Surgery, 525 East 68th Street, New York, NY 10021 (E-mail: jah9013@nyp.org).

J Thorac Cardiovasc Surg 2008;135:212-3

$0022-5223 / \$ 34.00$

Copyright $\odot 2008$ by The American Association for Thoracic Surgery doi:10.1016/j.jtcvs.2007.09.040

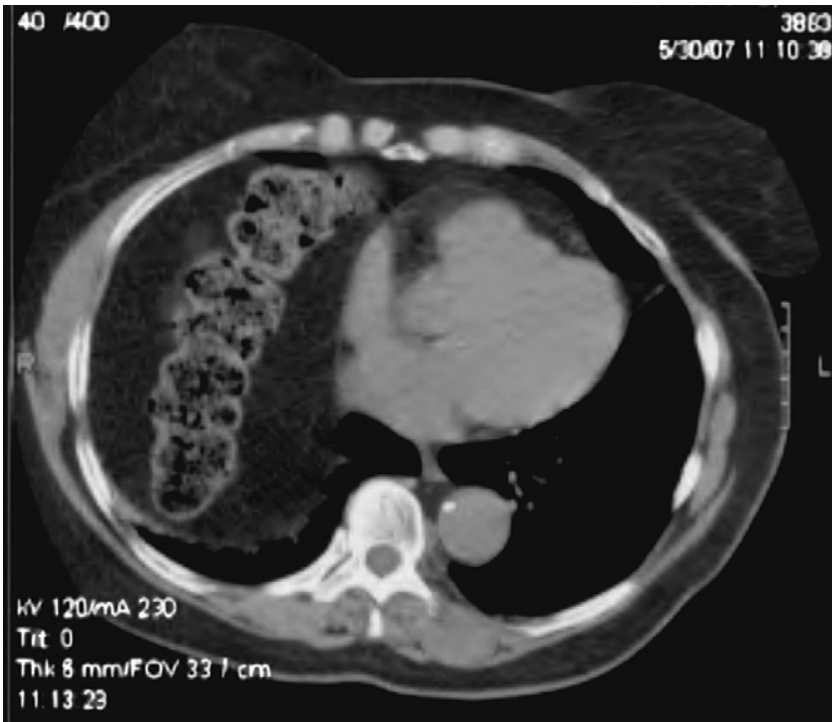

Figure 1. Computed tomographic scan demonstrating a large diaphragmatic hernia with air-filled colon and omentum in the right chest cavity.

NJ). The abdominal incision was closed using looped No. 1 absorbable sutures (Maxon; United States Surgical, Norwalk, Conn).

Postoperatively, the patient's course was relatively unremarkable. She initially had some abdominal fullness, which resolved with return of bowel function, and dyspnea requiring supplemental oxygen, which resolved with diuresis. She was discharged on postoperative day 7 tolerating a regular diet and without oxygen therapy.

\section{Discussion}

Morgagni hernia is rare, often small, and usually asymptomatic into adulthood. First described by Morgagni in the 18th century, this true hernia defect results from a failure of the anterior pleuroperitoneal membrane to fuse with the sternum and costal cartilages during embryogenesis. ${ }^{1,2,4}$ Other congenital abnormalities, including heart disease, gastrointestinal tract deformities, and Down syndrome, are associated with this hernia type. ${ }^{2,4}$ Morgagni hernia is more common in females and on the right side $(90 \%))^{2,4}$ Progressive enlargement of the anatomic defect may lead to clinical symptoms, often resulting from compression of thoracic organs or abdominal viscera within the hernia sac. Transverse colon and omentum herniate most frequently. ${ }^{1}$

Diagnosis of Morgagni hernia is usually made radiographically. ${ }^{2}$ Because most individuals are asymptomatic, a chest radiograph revealing an anteromedial mass is a common initial finding. ${ }^{1,2,4}$ Upper 


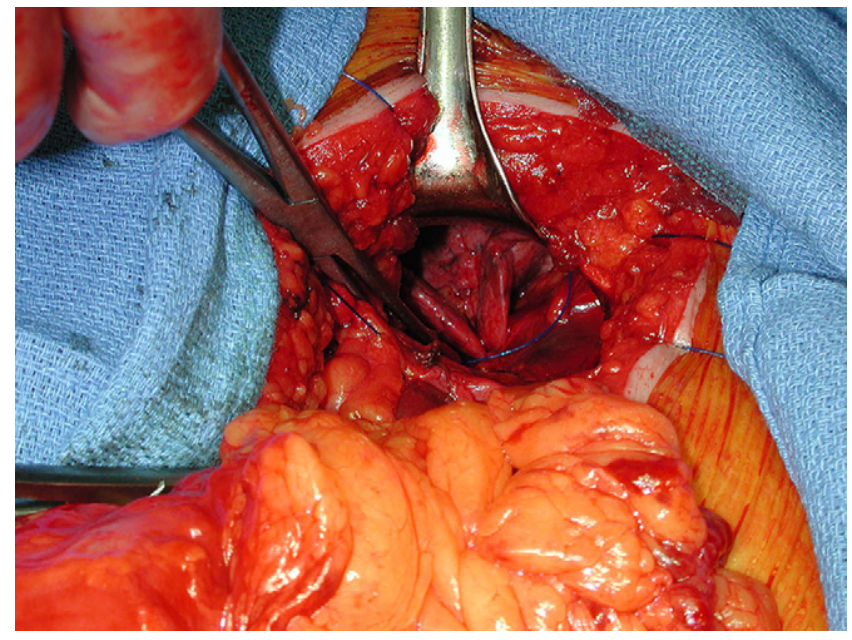

Figure 2. Laparotomy. Exploration revealed the entire transverse colon and omentum in the right chest cavity. After reduction of the hernia contents (bottom), the diaphragmatic defect and right lung are visualized.

gastrointestinal series or barium enema may reveal an opacified stomach, a small intestine, or a colon above the diaphragm. A review of the literature suggests that computed tomography scan is the best diagnostic tool, particularly for symptomatic hernias with potential incarceration and strangulation. ${ }^{5}$

Surgery is recommended for all diaphragmatic hernias. Either a transthoracic or transabdominal approach is acceptable. Some authors suggest that chronic hernias may be better suited to a transtho- racic approach secondary to extensive adhesions between the lung and the hernia, although there are insufficient data to support this claim. In addition to conventional open repairs, laparoscopic approaches have been tried with good success in the last decade..$^{3-5}$ We elected to perform a small upper midline laparotomy because it is difficult to lyse extensive adhesions between the hernia sac and the right lung, and then reduce a large hernia containing the entire transverse colon and greater omentum, via a relatively small hernia defect.

Intraoperative management of these hernias includes reduction of the herniated organs, identification and excision of the hernia sac, and closure of the anatomic defect. Similar to the principles of hernia repair in other locations, a tension-free repair is optimal. Primary closure with suture, primary repair with mesh reinforcement, and mesh closure alone are all viable options. Although most series in the literature are small, it seems that with proper diagnosis and surgical technique, these uncommon but potentially dangerous hernias can be treated with minimal morbidity and mortality.

\section{References}

1. Akhavan-Heidari M, Edwards D, Besenhaver J, Wolfer R. Incidental finding of congenital thoracic malformations in adult population. South Med J. 2006;99:539-43.

2. Rogers FB, Rebuck JA. Case report: Morgagni hernia. Hernia. 2006;10: 90-2.

3. Contini S, Dalla Valle R, Bonati L, Zinicola R. Laparoscopic repair of a Morgagni hernia: report of a case and review of the literature. J Laparoendosc Adv Surg Tech. 1999;9:93-9.

4. Yavuz N, Yigitbasi R, Sunamak O, As A, Oral C, Erguney S. Laparoscopic repair of Morgagni hernia. Surg Lap Endosc Percutan Tech. 2006; 16:173-6.

5. Swain JM, Klaus A, Achem SR, Hinder RA. Congenital diaphragmatic hernia in adults. Semin Laparosc Surg. 2001;8:246-55. 